\title{
Assessment of TerraSAR-X Products with a New Feature Extraction Application: Monitoring of Cylindrical Tanks
}

\author{
Raffaella Guida ${ }^{\mathrm{a}}$ IEEE Member, Antonio Iodice ${ }^{\mathrm{b}}$ IEEE Senior Member, \\ Daniele Riccio $^{\mathrm{b}}$ IEEE Senior Member \\ ${ }^{\mathrm{a}}$ Surrey Space Centre \\ University of Surrey \\ Guildford, UK \\ r.guida@surrey.ac.uk \\ ${ }^{\mathrm{b}}$ DIBET \\ Università degli Studi di Napoli "Federico II" \\ Napoli, Italy \\ \{iodice, daniele.riccio\}@unina.it
}

\begin{abstract}
There is no doubt that retrieving observed scene features is one of the most interesting and challenging activities in all fields of remote sensing: the successful extraction of scene parameters may not only mean the success of the adopted procedure, but often the success of a prediction model, of an image product, of a sensor project or, even, of an entire mission.

This paper is partly concerned with this. The mission, the sensor and the products at issue are the TerraSAR-X, the feature retrieval approach is the deterministic model-based approach already tested on E-SAR images and now in phase of improvement and testing on High Resolution (HR) TerraSAR-X images. Together with assessing the performances of TerraSAR-X products, this paper deals with a new application which till now has not received enough attention even if being worth of it: monitoring of big tanks in suburban or urban areas. Detailed discussion concerning the most suitable product for this kind of application is accompanied by retrieval results carried out on recently acquired TerraSAR-X images.
\end{abstract}




\section{Introduction}

As High Resolution (HR) SAR images have become available, new applications have become possible. But the approach able to disclose the truth contained in HR SAR images must be different from the previous ones. This is a general consideration, but it is particularly valid for SAR images of man-made structures. Indeed, the sizes of artificial objects are much closer to the present radar resolution: from streets and cars to buildings with elements like windows or balconies, urban scenarios need high resolution to let features be detected and monitored.

Obviously, together with the proper resolution, the proper product is required. Working with single look complex (SSC) products or geocoded products, in the azimuth-slant range plane or in the azimuth-ground range plane, might lead to different results. This happens because any kind of postprocessing performed on the SSC SAR image, even the simplest, introduces distortion due to operations like interpolation or sampling. In some cases, depending on the retrieval approach, the error introduced by these elaborations in the extraction process is acceptable or, at least, is a good price to pay in change of a more manageable product. Of course, this kind of consideration may change with the application at issue.

But appropriate resolution and product are nothing without the right approach. Today, with regard to urban scenes, we are more and more interested in punctual and deterministic extraction of the parameters of interest. The deterministic model-based approach, whose overall theory has been presented in [1-2] and which has been previously tested in the extraction of geometric and electromagnetic parameters from E-SAR images of urban scenes [3-4], is able to achieve high performance in feature extraction: geometric features are retrieved with an error often smaller than the image resolution [3] and, for the first time in literature, as far as we know, a building wall material is not simply foreseen but its dielectric constant is retrieved applying this approach [4]. These results lead also to the following consideration: an approach may be so powerful to suggest itself new applications. And this is the case of the present paper. 
In previous works on urban area monitoring with SAR images, attention has been mainly focused on buildings whose shape is approximately that of a parallelepiped [3-9]. In this paper, instead, we focus on big tanks, often present in the closest neighborhoods of urban centers, whose shape is that of a classical cylinder or that of a cylinder with a conic-shaped roof.

The interest for this kind of man-made structures arose for different reasons. Above all, the content of these tanks is often dangerous, being easily flammable (fuel, hydrogen, other kind of gases). Hence the need of their surveillance arises, especially in areas where climate or vegetated areas make the propagation of fires easy.

Moreover, differently from buildings, which can greatly vary in terms of dimensions, shapes and materials from country to country and, sometimes, even from city to city in the same country, this kind of structure is everywhere the same: for safety and logistic reasons, the best way to store gases and liquids is in cylindrical tanks and these structures are everywhere adopted.

Up to now this kind of structures has not received much consideration and nothing can be found in literature about them since the attention of scientific community was mostly devoted to understand and retrieve building structures in SAR images.

In this paper, we retrieve the height of some cylindrical tanks appearing in TerraSAR-X [10] images of the area of Naples from their brightness on the image, by using the model-based deterministic approach of $[1,3]$. Geometrical methods are also used for comparison purposes. An analysis of the suitability of different kinds of SAR products for the considered application is finally performed.

The paper is organized as follows: Section II contains a qualitative description of how cylindrical structures interact with the radar signal and, consequently, how they appear in SAR images; Section III details how the deterministic model-based approach, developed for parallelepiped-like structures, can be adapted to cylindrical structures; in Section IV TerraSAR-X images are analyzed with the above approach and retrieval results are presented together with discussion about the best product for the application at issue. Final comments and future perspectives are given in the Conclusions. 


\section{SAR images of cylindrical tanks: geometry}

The tanks that we propose to detect in the SAR images are cylinders large in terms of the employed resolutions. Sometimes their roof is not flat but exhibits a conic shape. In most cases, it is not fixed but floating to let the inner volume of the overall structure increase or decrease according to the content. For this kind of tanks, monitoring changes in the roof height leads to monitoring changes in the amount of the tank content

In this paper we focus on how standard cylindrical structure would appear in a SAR image. This is a structure of canonical shape, whose appearance in SAR images is relatively frequent.

Let us consider the cylinder in Fig.1. Let us suppose that it is placed on a rough terrain and its lateral surface is smooth also with reference to the employed electromagnetic wavelength. Each section at constant azimuth, obtained by cutting the cylinder with a plane parallel to the plane $y-z$, exhibits a rectangular shape and can be well treated as known in literature, see [2]. In practice, the contributions appearing in the SAR image at fixed azimuth are, in order from near to far range [11]: layover, double reflection, backscattering from the roof, triple scattering, shadow, see Fig.2. Similarly to the case of the parallelepiped (which is usually the appropriate model to deal with an edifice), each section of this cylinder is rectangular; but, differently from it, each section is different from its neighboring sections and is localized in a different point. For example, the section obtained with a tangent plane in $x=x_{0}$, see Fig.1, degenerates from a rectangle into a segment whose contributions in the SAR image are the layover and double reflection soon followed, along the slant range direction, by shadow.

A preliminary visual inspection (full discussion is deferred to the next sections) of a SAR image of a tank leads to identify more contributions than those expected and predicted by the above mentioned model. In the SAR image in Fig.3a, where the illumination comes from the right side, contributions from the borders of the roof are visible. In this paper we do not deeply discuss the nature of these contributions since it involves scattering mechanisms different from the reflection 
and scattering, and somehow described by diffraction phenomena whose application to SAR images is completely new and is at the moment under study by the Authors of this paper: this will be the subject of a future work. However, we want here to underline how useful they can be in some cases: in fact these contributions are aligned along a direction perpendicular to the radar flight trajectory which in this way can be immediately retrieved in many SAR products where it was lost due to rotation operations (as in geocoded products). The sequence of other contributions will discriminate between left or right illumination.

In Fig.3b the red arc highlights the double reflection curve. Let us underline that the ground has been supposed to be rough at the wavelength scales: this assumption leads to double reflection contributions also for rays whose incidence on the cylinder lateral surface takes place at any $x \neq x_{c}$. These rays are reflected towards the ground and then scattered back toward the radar. Obviously, the larger the distance between $x$ and $x_{c}$, the smaller the intensity of double reflection contribution as predicted by the theory of electromagnetic scattering [12].

In Fig.3c, the moon-shaped region (between the right side of the backscattering from the upper border and the double reflection curve, whose borders in the SAR image are highlighted in green) corresponds to the layover area, which includes the backscattering from ground, lateral surface and roof. The region between the double reflection curve and the left side of the backscattering from the upper border is characterized by both the backscattering from the roof and triple reflection (wallterrain-wall) contribution. Finally, at larger slant range coordinates, a shadowed area follows.

Retrieval of geometric shape of this cylinder and its dimensions is quite simple: assuming a circular shape for the cylinder base, the base diameter $D$ and the height $h$ of the lateral surface are the only quantities to be extracted from the SAR image. The diameter $D$ can be retrieved first measuring in the SAR image the length of the segment $A C$, see Fig.3d:

$D=A C / \sin \vartheta$

$D=A C$
(1.1), for images displayed in the slant range-azimuth plane

(1.2), for images displayed in the ground range-azimuth plane 
where $\theta$ is the radar look angle and the distance $A C$ is the number of pixels along the segment $A C$ times the pixel spacing in range; note that in the SAR image, this distance can be easily determined because $A$ and $C$ are clearly visible. In case of single look complex data, the segment $A C$, which is along the slant range, is aligned with the image abscissa (this is the case of Fig.3d). In this case, the extraction of $D$ may be even simpler by measuring along the azimuth direction the distance $P Q$ in pixels and multiplying it by the azimuth resolution. In geocoded products, instead, being the image rotated, $A C$ is not aligned with the image abscissa and can be computed as

$$
A C=\sqrt{y_{A C}^{2}+x_{A C}^{2}}
$$

where $y_{A C}$ and $x_{A C}$ are, respectively, the projection of AC along the image ordinate and abscissa. As regards the cylinder height, we have, see Fig.3d,:

$$
\begin{array}{ll}
h=B C / \cos \vartheta & (1.4), \text { for images displayed in the slant range-azimuth plane } \\
h=B C \tan \vartheta & (1.5), \text { for images displayed in the ground range-azimuth plane }
\end{array}
$$

where the distance $B C$ is estimated by the number of pixels along the segment $B C$ times the pixel spacing in range. $B C$ is, whatever the image plane, the distance between the brightest point belonging to the double reflection line and the right (or left) brightest point of the upper edge for right (or left) illumination.

This structure holds very valuable properties; its canonical shape can be easily represented in an ad hoc geometrical model, and its appearance in actual SAR images is certainly not rare: this suggests us to adopt sites containing tanks to:

- Compare results relative to the same feature extracted by different products, using the couples of equations (1.1),(1.4) or (1.2),(1.5) to assess the best TerraSAR-X product for geometric features retrieval from geometric parameters, performed in Section IV; 
- Further test new algorithms developed for buildings, such as geometrical features retrieval from electromagnetic parameters [1, 3], according to the theory explained in Section III. Results are all presented in Section IV.

\section{SAR images of cylindrical tanks: electromagnetics}

The approach that we adopt to study these structures is based on the methods developed in [1-4] for usual parallelepiped-shaped buildings. Accordingly, we hereafter briefly recall the approach developed in [1-4], and then we detail how it may be adapted to cylindrical structures presented in Section II.

The radar cross section of the dihedral structure formed by a building vertical wall and rough soil can be expressed as

$$
\sigma^{\circ}=h \cdot f(\mathbf{p})
$$

where $\mathbf{p}$ is a vector of known parameters: $\mathbf{p}=\left(l, \sigma, L, \varepsilon_{w}, \varepsilon_{s}, \varphi, \vartheta\right)$ with $l$ building length, $\sigma$ and $L$ standard deviation and correlation length of the stochastic process describing the soil roughness, $\varepsilon_{w}$, $\varepsilon_{s}$, complex dielectric constant of the walls and soil, respectively. Indicated with $S_{p q}$ the scattering matrix, with $p$ and $q$ each standing for $\mathrm{H}$ (horizontal) or $\mathrm{V}$ (vertical) polarization, see [1-2] for complete expressions, $f(\boldsymbol{p})$ is given by either

$$
f(\mathbf{p})=\left|S_{p q}\right|^{2} l \tan \vartheta \cos \varphi \exp \left(-4 k^{2} \sigma^{2} \cos ^{2} \vartheta\right) \sum_{m=1}^{\infty} \frac{(2 k \sigma \cos \vartheta)^{2 m}}{m !} \frac{k^{2} L^{2}}{4 m} \exp \left[-\frac{(2 k L \operatorname{sen} \varphi \operatorname{sen} \vartheta)^{2}}{4 m}\right]
$$

if Geometric Optics - Physics Optics (GO-PO) solution is considered for the double scattering incoherent radar cross section $\sigma^{o}$, or 


$$
f(\mathbf{p})=\frac{\left|S_{p q}\right|^{2} l \tan \vartheta \cos \varphi\left(1+\tan ^{2} \vartheta \sin ^{2} \varphi\right) \cdot \exp \left[-\frac{\tan ^{2} \vartheta \sin ^{2} \varphi}{2 \sigma^{2}\left(2 / L^{2}\right)}\right]}{8 \pi^{2} \cos ^{2} \vartheta \cdot \sigma^{2}\left(2 / L^{2}\right)}
$$

when GO-GO solution is assumed for the double scattering radar cross section $\sigma^{\circ}$.

The height of a building can be then obtained by measuring the gray level of the building doublereflection bright line on SAR image and then inverting eq.(3.1). If the SAR image is not radiometrically calibrated, unknown multiplicative and additive constants can be determined by using at least two buildings of known height in the scene as calibrators, as described in [1].

We do not deepen this theory in this paper: our aim is here to assess the feasibility of TerraSAR-X products, dealt with in the next section, and show a further application that can be simply derived by that developed for the buildings in [1,3]. More details on the theory and on the retrieval algorithm are reported in $[1,3]$.

Let us now move to consider how the above described method can be applied to cylindrical structures. In general, the intensity of cylinders in SAR images can be explained by employing appropriate backscattering models. The exact solution in closed form for this electromagnetic problem can only be provided under some approximations; the corresponding solutions can then be found in the literature and should be somehow adapted to cope with the requirements of this specific problem. In general, we can identify two limiting cases:

1. cylinders whose dimensions are much smaller than the resolution cell, $D<<\Delta y, \Delta x$;

2. cylinders whose dimensions are much larger than the resolution cell, $D>>\Delta y, \Delta x$,

where $D$ is the cylinder diameter and $\Delta y, \Delta x$ are the dimensions of the resolution cell, respectively, in ground range and azimuth.

Considering the resolution cell of the TerraSAR-X products that, but for the scansar mode, ranges from about $3 \mathrm{~m} \times 3 \mathrm{~m}$ relevant to the stripmap mode to $1 \mathrm{~m} \times 1 \mathrm{~m}$ relevant to the spotlight mode [10], 
we can simply assess that the tanks dimensions satisfy the above mentioned condition 2 , because their height and diameter are usually greater than tens of meters. With the high resolution SAR images only urban objects like electricity poles may satisfy the condition 1 .

The above mentioned distinction underlines that the approach and models to study the effect of cylinders shape on the SAR image change according to the resolution: this concept is elucidated hereafter. Models that satisfy condition 1 can be easily found in the literature because many studies have been carried on to attempt a reasonable description of the electromagnetic field backscattered by single curved surfaces, see for instance [12-13]. Involved expressions for the backscattering derived for different cases, indefinite cylinders or cylinders with finite dimensions, made of perfect electric conductors or dielectric material, in oblique or specular incidence, can be found in [13]. Those expressions are analytical, having been evaluated solving Maxwell's equations for the case at issue. But, unfortunately, they are not very friendly. The presence of Bessel and Hankel functions allows a closed form expression for the backscattered field in terms of the radar and scene key parameters. However, unless we make some approximations [13], these functions require use of numerical codes to be evaluated, the control on the relative dependence among parameters is lost and, consequently, the possibility of inverting expressions for retrieving information about parameters, once that the backscattered field was measured on the image, is also lost. Moreover, the presence of the ground is never considered and, consequently, also the possible multiple reflections that may arise. But, at the moment, no alternatives exist for this case and, luckily, the structures involved are not of particular interest yet.

Also in the case of cylinders whose dimensions are much larger than the resolution cell, we are interested in looking for analytical closed-form expressions of the field backscattered by this kind of structures: in this case the approach may hopefully bring to simple direct scattering solutions that lead to successful inversion frameworks. To this aim we tried to apply the approach in [2] to a 
single curved surface, obviously assuming the same hypotheses employed in [2] for the ground (rough) and the cylinder surface (smooth).

The curved lateral surface of the cylinder can be discretized at the SAR resolution scale so that for each cylinder many flat vertical planes, which can be treated as the building frontal wall in [2], are individuated. In other words, the cylinder is approximated by an appropriate irregular prism, as detailed hereafter. Let us consider Fig.4a, in which the base of a cylinder is represented and a resolution cell grid is superimposed. In the $x-y$ plane, the curve is approximated in the $i$-th resolution cell by its tangent in the middle point $\left(x_{m i}, y_{m i}\right)$; conversely, in the $x-y-z$ space, each elemental curved surface is approximated by its tangent plane in its middle vertical line. This plane is here treated like the building frontal wall in [1-4]. Use of this approximation deserves some comments.

On one side, obviously, the better the resolution, the better the approximation that we get by employing the prism instead of the actual cylinder. Moreover, if the base of the cylinder does not occupy a large number of resolution cells, it turns out that the irregularity in approximating the cylinder increases, which might lead to predict an artificial irregular behavior on the radar image (see, for example the visual difference exhibited by cells 1 and 3 in Fig.4b). In terms of scattering parameters this provides a not uniform variation, from cell to cell, of the angle $\varphi$ between a generic tangent at the curve (representing the approximating prism) and the radar flight trajectory. Physically, this may cause the coherent sum of much different values of the field in the cells where a large variation of the actual incident angle is expected; as a matter of fact, the dependence upon $\varphi$ for each kind of contribution to the scattered field may be critical $[2,4]$. Following this consideration, products relative to data acquired in the high spotlight mode should be preferred to those acquired in the stripmap geometry.

On the other side, some measurements carried on with our approach [1,3-4] are based on averages over many pixels to reduce the speckle effect. A proper example is the building height retrieved 
from the brightness of the double reflection contribution $[1,3]$. In those papers the building shape is represented as a parallelepiped and the building height, being the same in any vertical section, could be, at least in principle, retrieved by measuring the brightness of any pixel belonging to the double reflection line. In practice, the whole double reflection line can be conveniently used and the brightness of all pixels of the line can be averaged for a better estimation of the building height. Conversely, for cylindrical shapes, curved actual walls basically complicate this averaging since now the double reflection contribution is distributed along a curve and a smaller number of pixels should be employed. This again leads to prefer the highest resolution products.

In summary, the above reported considerations on the cylinder shape discretization allow to conveniently and safely apply an appropriately modified version of the theory on building height estimation that we developed and presented in [1,3]. The extraction of cylinder height from the double reflection contribution will be carried on in the next section on different kinds of SAR products and the steps performed, for each cylinder, are the following:

- detection of the double-reflection curve produced by the frontal curved surface and the rough ground (this detection is here performed manually, but very simple automatic edge detection algorithms can be used as well);

- the brightest pixel of the double-reflection curve is simply extracted. Its grey level is averaged with those of its closest neighbours;

- as in $[1,3]$, this value is directly linked to the height of the structure in that section by inverting eq.(3.1).

In the next section, by using different TerraSAR-X products, the dimensions of cylinders are retrieved from geometric parameters measurable on the SAR image as explained in Section II while the height is also retrieved from radiometric parameters exploiting the idea of discretization presented before and adapting a modified version of the approach in $[1,3]$. 


\section{Tank feature retrieval from TerraSAR-X images}

In this section we compare results of tanks features extraction obtained by applying the approaches previously explained to three different kinds of TerraSAR-X data products:

1. Enhanced Ellipsoid Corrected (EEC) product for data acquired in the stripmap mode;

2. Single look Slant range Complex (SSC) product for data acquired in the stripmap mode;

3. SSC product for data acquired in the high spotlight mode.

This choice of products and acquisition modes is aimed at better investigating:

- The influence of post-processing (SSC vs. EEC) on feature extraction from geometric and radiometric parameters: products 1 and 2, in fact, are relevant to data acquired at the same time, on the same scene and in the same configuration mode;

- The influence of configuration modes (High Spotlight vs. Stripmap) on feature extraction from geometric and radiometric parameters: both products 2 and 3, in fact, have not been post-processed, but they are relevant to data acquired in different configuration modes.

A brief description of products and operative modes relevant to the studied images follows together with the respective feature extraction results and comments.

\section{IV.1 Enhanced Ellipsoid Corrected (EEC) - Spatially Enhanced (SE) - Stripmap (SM)}

According to the description of products in [14], EEC is a multi look detected product derived by projecting and re-sampling the original data to the WGS84 reference ellipsoid. The image distortions caused by varying terrain height are corrected by using an external Digital Elevation Model (DEM). The achievable accuracy in pixel localization depends on the orbit data precision, the timing accuracy and specifically the elevation accuracy of the reference DEM.

In a spatially enhanced product, [14], the highest possible square ground resolution is achieved. The smaller (between azimuth and range) resolution value is adjusted to the larger one and the corresponding reduction of the bandwidth is used for speckle reduction. 
Finally, the image has been acquired in the standard stripmap mode, in which the antenna beam direction is perpendicular to the radar flight trajectory and fixed along all the acquisition time [15]. The EEC_SE_SM product we analyze was acquired on the area of Naples, Italy, on19th March 2008.

The main operating radar parameters are in Table I, column 2, where image resolutions and pixel spacings are also given.

\section{IV.2 Single Look Slant Range Complex (SSC) - Stripmap (SM)}

This product is the basic single look product of the radar signal, given in complex representation, [14] and refers to the same acquisition and scene presented in EEC. The plane of the image is in azimuth - slant range. The main operating radar parameters are in Table I, column 3, where image resolutions and pixel spacing are also given.

\section{IV.3 Single Look Slant Range Complex (SSC) - High resolution Spotlight (HS)}

The main difference of this product regards the acquisition mode, which is now spotlight. In this configuration, during the acquisition time the antenna beam steers along a fixed point in the scene thus increasing the length of the synthetic aperture and improving the azimuth resolution, [15]. These data have been acquired on the area of Naples, too, on $28^{\text {th }}$ July 2008 , with radar parameters similar to those of the previous products except for a larger look angle, see Table I, column 4 . The better values of resolution are evident.

\section{IV.4 Description of the site}

The study area is in the close neighborhoods of the center of Naples and presents a large concentration of big tanks. Relevant cartography and an aerial picture are reported in Fig.5, (a) and (b), respectively. According to a recent study of feasibility [16], promoted by the Italian Ministry of Infrastructures, regarding industries at risk of considerable incident in the province of Naples, this area has been marked as a high risk one. Not far, similar areas can be found too and all are really dangerous, the tanks containing oil, sulphuric acid, fluoridric acid, toluene, pentan and other 
chemical products for industry which are extremely toxic and detrimental to the environment [16]. The high danger represented by the content of the tanks, as previously said, suggested us to study how to monitor this kind of structure by using TerraSAR-X images.

The company owner of the tanks provided us with the information relevant to the dimensions of the four tanks marked with numbers 1-4 in Fig.5, and some visits to the site allowed us verifying that the ground conditions (in term of roughness and material) are the same for all the soils surrounding the tanks. These conditions, in fact, allow to simplify the procedure of the height extraction from double reflection contribution [1]. The ground truth of the four tanks is provided in Tables II and III, respectively for the diameter and the height.

\section{IV.5 Results}

The analyzed images are in Fig.6, Fig.7 and Fig.10, respectively for the EEC_SE_SM, SSC_SM, SSC_HS. We can simply understand by Fig.6 that the EEC product may be not adequate for most of features extraction, either it is carried on geometrical parameters (straight lines in the raw signal wrongly appear like curves because of projection on DEM, see and compare Fig.6 and Fig.7) or radiometric parameters (re-sampling also occurs). For the case at issue we tried the extraction of $D$ and $h$ obtaining very different results, see Tables II and III respectively, "EEC Stripmap" columns. In fact, while the height is retrieved in three cases with an error not much larger than the resolution, the extraction of $D$ seems to be completely wrong: in one case the error is even about $1 / 3$ of the right value. We will see later that this error is due only in part to the kind of product. In fact, should it be related only to the product, it would disappear in the measurements on SSC while it is present also there.

The height extraction based on double reflection was impossible to perform with this product because of the unreliability of the image radiometry for the proposed approach.

When we work on SSC product, Fig.7 and Tables II, III, "SSC Stripmap" columns, the results are much better and, as we can see from the tables, more different measures can be carried out. Above all, the diameter can be retrieved also from the azimuth direction and the results are now 
reasonable and, more importantly, the errors are smaller than the azimuth resolution $(3.00 \mathrm{~m}$ for the SSC, see Table I, column 3), while the extraction carried on along the range is still unsuccessful. The reason of the discrepancy in the two extractions of $D$ (those performed by employing range or azimuth directions, respectively) was simply found in the presence of a floating roof. This structure left also a clear signature in the SAR image in the presence of concentric circles of points which are related to its metallic framework, see Fig. 8 . Now the presence of a floating roof may create a further dihedral which in turn is responsible for another mechanism of double reflection. The effect is other bright points $\left(D_{2}\right.$ in Fig.10) not far in range from the scattering from the upper edge of the lateral surface $\left(\mathrm{S}_{2}\right)$, but brighter. When the illumination comes from the left side, as in our images, see Fig.9, this further double reflection contribution called $\mathrm{D}_{2}$ is placed at the right side of the scattering from the upper edge of the lateral surface $\left(\mathrm{S}_{2}\right)$, which explains why $D$ is always overestimated when retrieved from the slant range (the error $e_{D}=D_{\text {retrieved }}-D_{\text {true }}$ is always positive). In practice, a worsen accuracy in the localization of the pixels of interest occurs. However, it is important to note that, if the above interpretation is correct, from the difference between the $D$ values retrieved along the range and azimuth directions it is possible to retrieve the (time-varying) height of the floating roof, which can be considered a measure of the volume of liquid stored in the tank.

The interesting additional possibility in this SSC case is the retrieval of the tanks height exploiting the radiometry of the image, more precisely the brightness of the double reflection contribution to the radar cross section according to the approach and the guidelines presented in Section III. The heights of two tanks have been supposed to be known for the operation of calibration [1], but the heights of the other two tanks have been retrieved with an error that is much smaller than that presented by the height retrieval from geometry. This is a very good result, assessing the quite total independence of this kind of measure from the spatial resolution of the image and the efficiency of the adaption of an approach at first developed for a completely different geometric structure. We also note that, again, the effect depicted in Fig.9 can be used to retrieve the height of the floating roof (and hence monitoring the volume of liquid stored in the tank) also by 
using the brightness of the further double reflection point (i.e., $\mathrm{D}_{2}$ in Fig.9) provided that a further better resolution is available.

The goodness of the above results is further confirmed by the measurements performed on the SSC_HS product, see Fig.10 and Tables II,III, "SSC Spotlight” columns. Only for the SSC_HS, the heights of two other surrounding tanks, those with numbers 5 and 6 in Fig.10, have been extracted by double reflection contribution. These tanks present conic-shaped roofs, a difference in the geometry which does not affect particularly the approach. The ground truth relevant to these structures has not been provided but, from the shadow they project on the ground in the optical image, see Fig.5b, is possible to deduce that their heights are not very different from the others and, consequently, with high probability, the extraction presents an error smaller than 1 meter.

As regards the geometrical extractions, also here $D$ is better retrieved from the azimuth than from the range, even if now the error in the extraction of $D$ from range is smaller than the one presented by the same extraction performed on the SSC_SM because of the better spatial resolution. Consistently, also the height extraction from the geometry is better than in the other products.

Overall comments on the suggested application and its exploitation of high resolution in TerraSAR-X products are given in the Conclusions.

\section{Conclusions}

In this paper we introduce the possibility to estimate some geometric features of an actual class of man-made objects with accuracy better, or sometimes much better, than the SAR resolution. In particular, we selected some cylinders that very frequently appear in the surroundings of urban areas and in the areas of main harbors. These cylinders are used as tanks for a series of dangerous and hazardous products and their monitoring in real (or almost real) time is largely advisable. It has been shown that the best kind of data to estimate the tanks geometric features are 
provided by very high resolution SAR sensors that on spaceborne platform are now operative within the TerraSAR-X mission.

In particular we showed that by inverting an appropriately modified scattering model, the height of the tanks can be retrieved with an accuracy better, even much better, than the SAR resolution by employing just one single SAR image; as a corollary, for tanks with floating roofs we are able to estimate the amount of content can be extracted. Being TerraSAR-X data acquired on regular basis, these results open the possibility to implement some applications for monitoring this sort of tanks also in favor of the civil protection. This characteristic of our approach is original and very favorable with respect to other approaches that are based on multiple images often acquired within a very large amount of time and multiple observation directions. The rationale is also different from others presented in the literature since we only make use of amplitude data, whereas use of phase differences between multiple images is usually employed to retrieve geometric features of deterministic or coherent objects in SAR images. Finally, in our approach the inversion of a scattering model is performed, whereas the extraction of geometric features is usually obtained by employing phase differences data that do not bring at all any scattering information after appropriate pre-processing techniques.

The approach we presented deals with actual amplitude data on an actual spaceborne SAR. Accordingly, in this paper we also detailed the effect of working on different amplitude images (products) provided by the TerraSAR-X sensor. In particular it has been shown that SSC images can be conveniently used and must be preferred for the extraction of the tanks heights, whose retrieved values present an error of a few centimeters compared to the ground truth, whereas an appropriate mix of existing products can be employed to obtain at the same time also the proper space location in lat-long coordinates.

Factors affecting tanks height estimation have been also analysed: within this framework, calibrating tanks, effect of speckle and SAR resolution have been discussed. The analysis carried on a controlled data set of TerraSAR-X images, employed along with a ground truth campaign, proved 
the accuracy of our results; in addition we were able to justify the different accuracy that we obtained under different data sets and operating conditions. These latter results were obtained thanks to the characteristics of our approach that is based on the inversion of a deterministic scattering model. As a matter of fact, the analytical inversion allowed by our direct model and the considerations on double reflection lines, relative calibration and products selection issues, lead us to fully consider our approach as a reliable inverse scattering model for retrieving the height and diameter (and, hence, the volume) of tanks from their backscattering signatures.

\section{Acknowledgements}

The authors thank DLR for providing us with the TERRASAR-X data and Kuwait Raffinazione e Chimica SpA for providing the ground truth relevant to the analyzed scene.

\section{References}

[1] R.Guida, A.Iodice, D.Riccio, "Retrieval of the Height of Isolated Buildings from Single High Resolution SAR Images", IEEE Trans. Geosc. Remote Sensing, submitted.

[2] G.Franceschetti, A.Iodice, D.Riccio, "A canonical problem in electromagnetic backscattering from buildings", IEEE Trans. Geosc. Remote Sensing, vol.40, pp.1787-1801, 2002.

[3] R.Guida, G.Franceschetti, A.Iodice, D.Riccio, G.Ruello, U.Stilla, "Building Feature Extraction via a Deterministic Approach: Application to Real High Resolution SAR Images", Proc. Geosc. Rem. Sens. Symp., pp.2681-2684, Barcelona (Spain), 2007.

[4] R. Guida, A. Iodice, D. Riccio, U. Stilla, "Model-Based Interpretation of High-Resolution SAR Images of Buildings", IEEE Journal of Selected Topics in Applied Earth Observations and Remote Sensing, vol.1, no.2, pp.107-119, June 2008. 
[5] M.Quartulli, M.Datcu, "Stochastic Geometrical Modeling for Built-Up Area Understanding from a Single SAR Intensity Image with Meter Resolution", IEEE Trans. Geosc. Remote Sensing, vol.42, pp.1996 2003, 2004.

[6] C.Tison, F.Tupin, H.Maitre, "Retrieval of building shapes from shadows in high resolution SAR interferometric images", Proceedings of the International Geoscience and Remote Sensing Symposium, pp.1788-1791, Toulouse (France), 2004.

[7] E.Simonetto, H.Oriot, R.Garello, "Rectangular building extraction from stereoscopic airborne Radar images”, IEEE Trans. Geosc. Remote Sensing, Vol. 43, pp.2386 - 2395, 2005.

[8] Feng Xu, Ya-Qiu Jin, “Automatic Reconstruction of Building Objects From Multiaspect MeterResolution SAR Images”, IEEE Trans. Geosc. Remote Sensing, Vol. 45, pp.2336 - 2353, 2007.

[9] A.Thiele, E.Cadario, K.Schulz, U.Thonnessen, U.Soergel, "Building Recognition From MultiAspect High-Resolution InSAR Data in Urban Areas", IEEE Trans. Geosc. Remote Sensing, Vol.45, pp.3583-3593, 2007

[10] S.Buckreuss, R.Achim, "Status Report on the TerraSAR-X Mission”, Proc. Geosc. Rem. Sens. Symp., pp.II-379-II-381, Boston (Massachusetts), 2008.

[11] G.Franceschetti, A.Iodice, D.Riccio, G.Ruello, "SAR Raw Signal Simulation for Urban Structures", IEEE Transactions on Geoscience and Remote Sensing, GE-41, 1986-1995, 2003.

[12] E. Knott et al, Radar Cross Section 2Nd Ed, Ed.Scitech, 2004.

[13] G.T.Ruck, D.E.Barrick, W.D.Stuart, C.K.Krickbaum, Radar Cross Section - Handbook, Vol.2, Ed. Peninsula Publishing, 2000.

[14] T. Fritz, M. Eineder, "TerraSAR-X Ground Segment Basic Product Specification Document”, http://www.dlr.de/tsx/documentation, 2008.

[15] C. Elachi, Spaceborne Radar Remote Sensing: Applications and Techniques, IEEE Press, New York, 1987.

[16] http://sit.provincia.napoli.it/home.asp 


\section{Captions of Figures}

Fig.1: Pictorial view of a cylinder based on a rough terrain.

Fig.2: Cut at constant azimuth of the scene in Fig.1 and relevant contributions in the SAR image.

Fig.3: Different contributions in the SAR image of a cylinder: (a) scattering from the upper edge; (b) double reflection; (c) layover; (d) representation of segments to be measured in the SAR image for the extraction of the cylinder dimensions from geometrical parameters.

Fig.4: Discretization: (a) a grid with a resolution cell step is superimposed at the cylinder base, (b) enlarged view.

Fig.5: Area under study: (a) relevant cartography, (b) aerial picture.

Fig.6: EEC_SE_SM image of the scene in Fig.5.

Fig.7: SSC_SM image of the scene in Fig.5.

Fig.8: Cylinders with floating roof: (a) typical contribution in a SAR image; (b) metallic infrastructure of the floating roof.

Fig.9: Pictorial representation of contributions superimposition in a SAR image of a cylinder with floating roof.

Fig.10: SSC_HS image of the scene in Fig.5. 


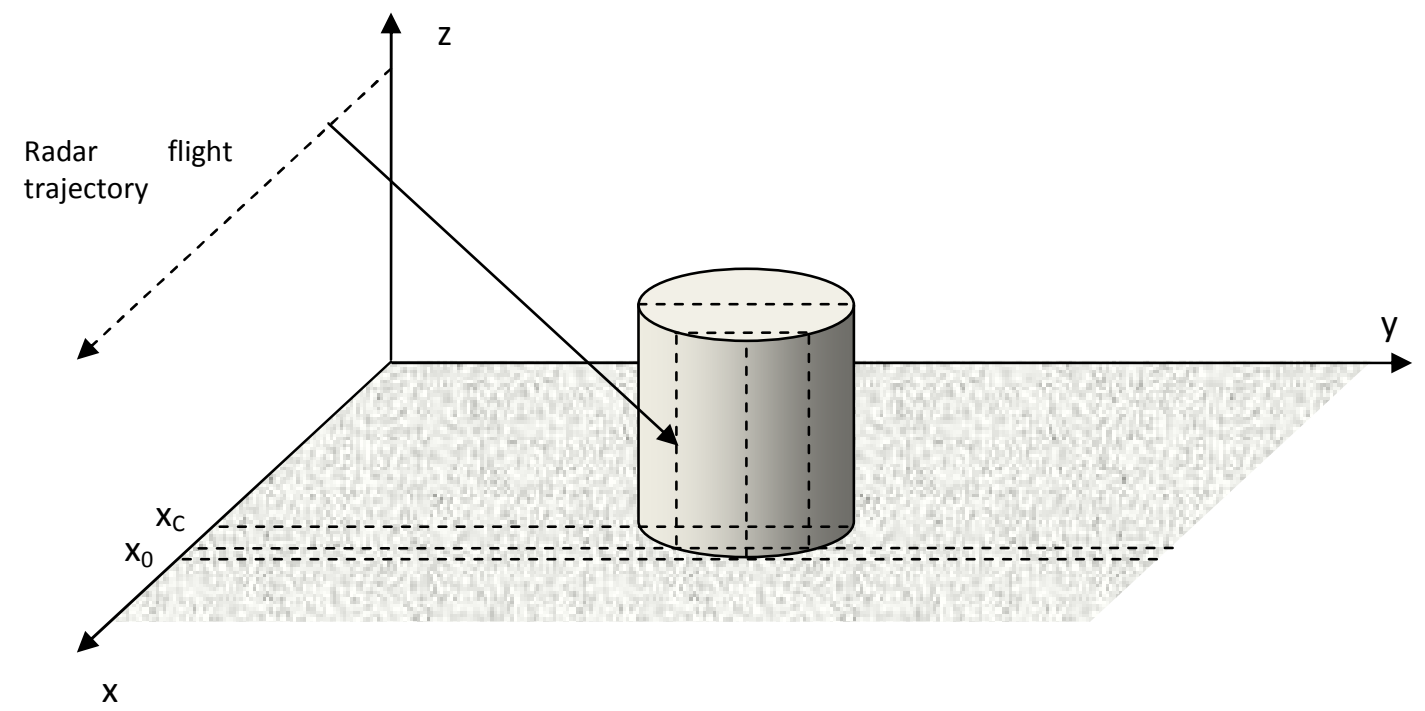

Figure 1

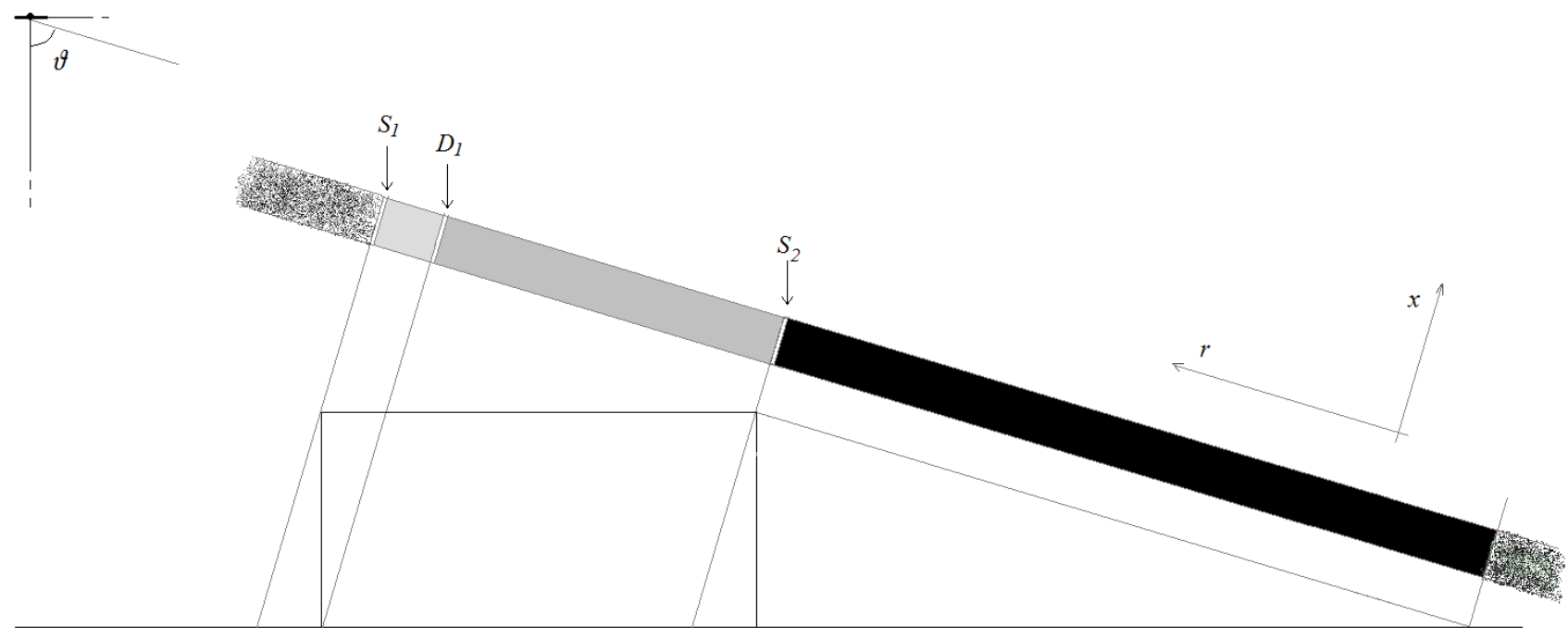

Figure 2 


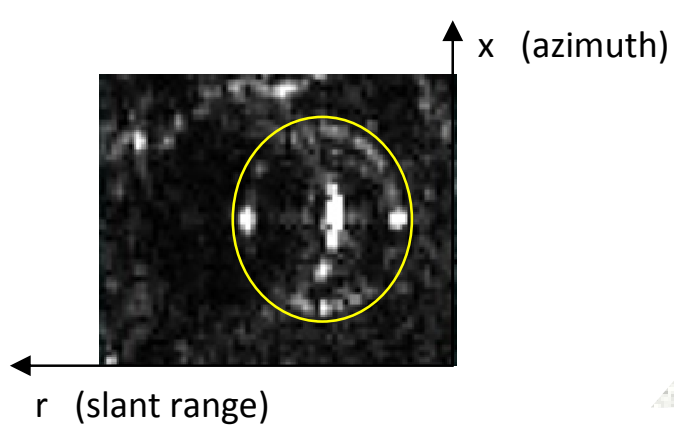

(a)

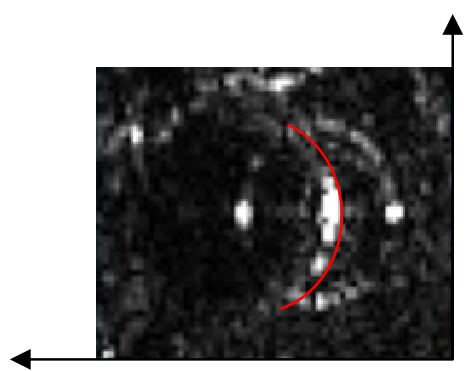

$r$ (slant range)

(b)

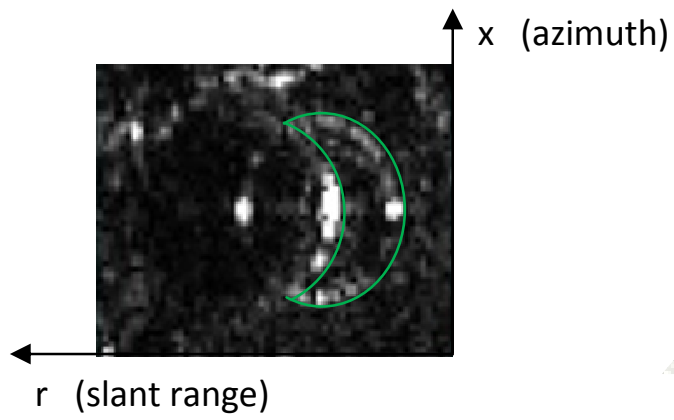

(c)

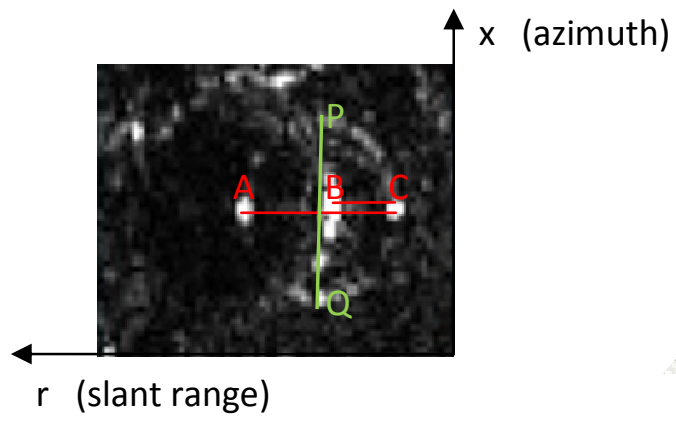

(d)

Figure 3 


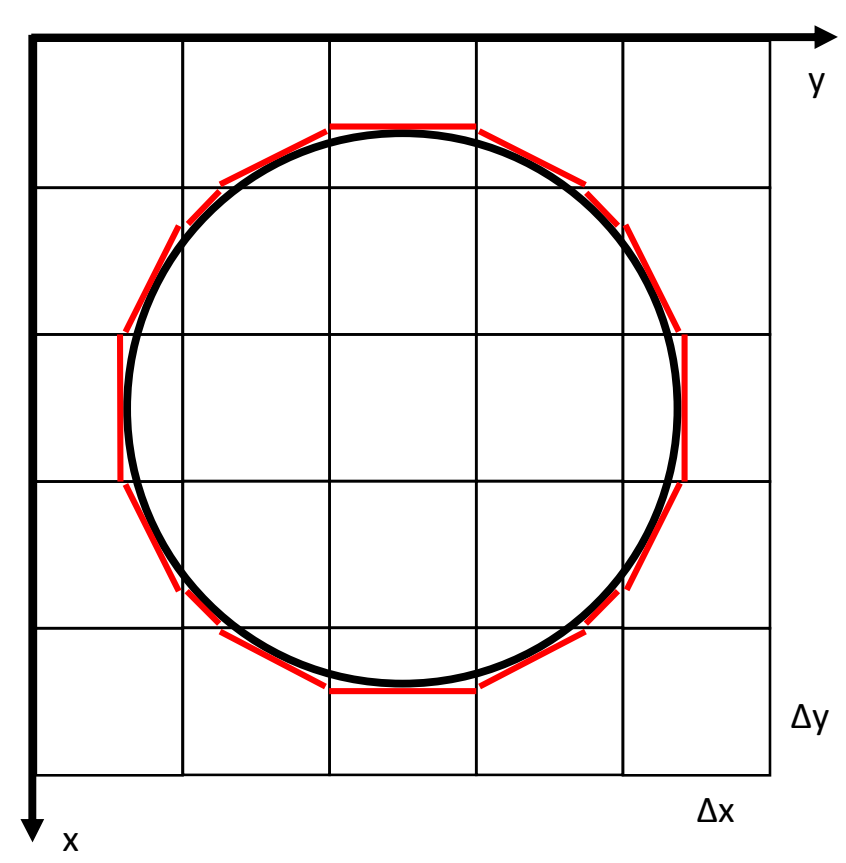

(a)



(b)

Figure 4

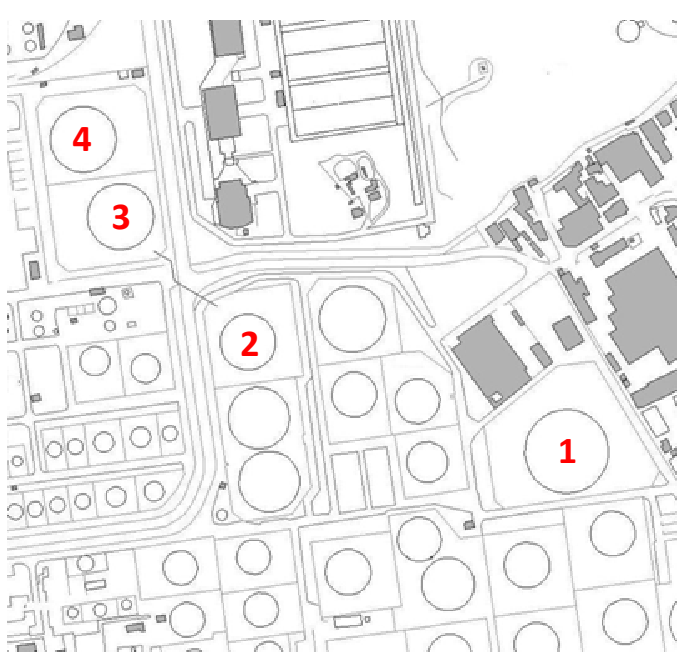

(a)

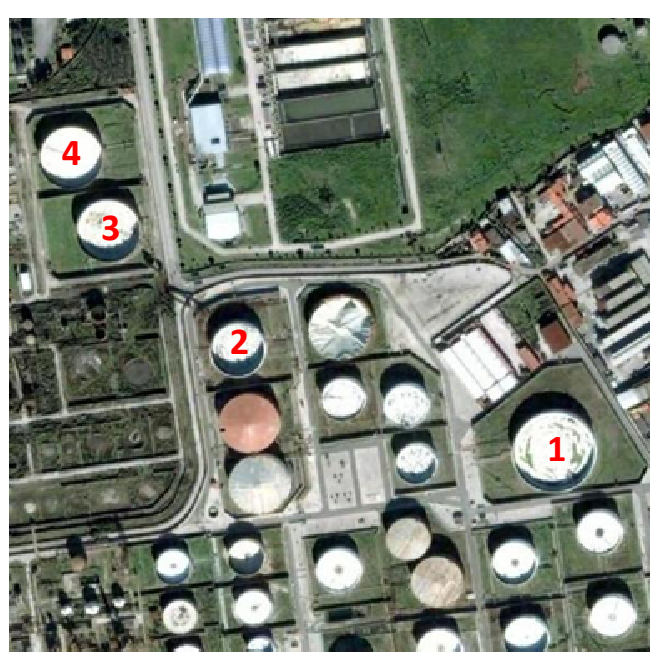

(b)

Figure 5 


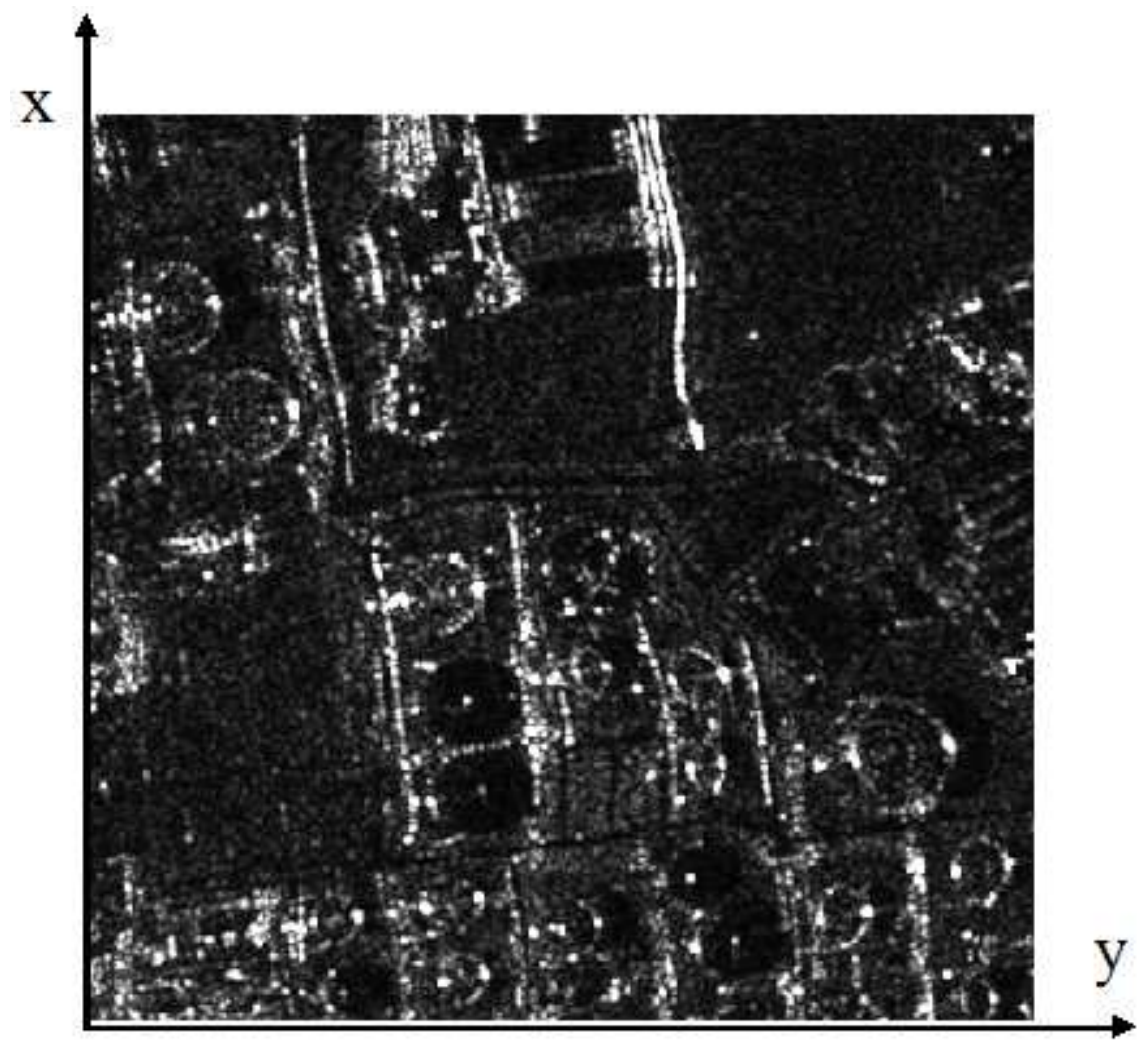

Figure 6 


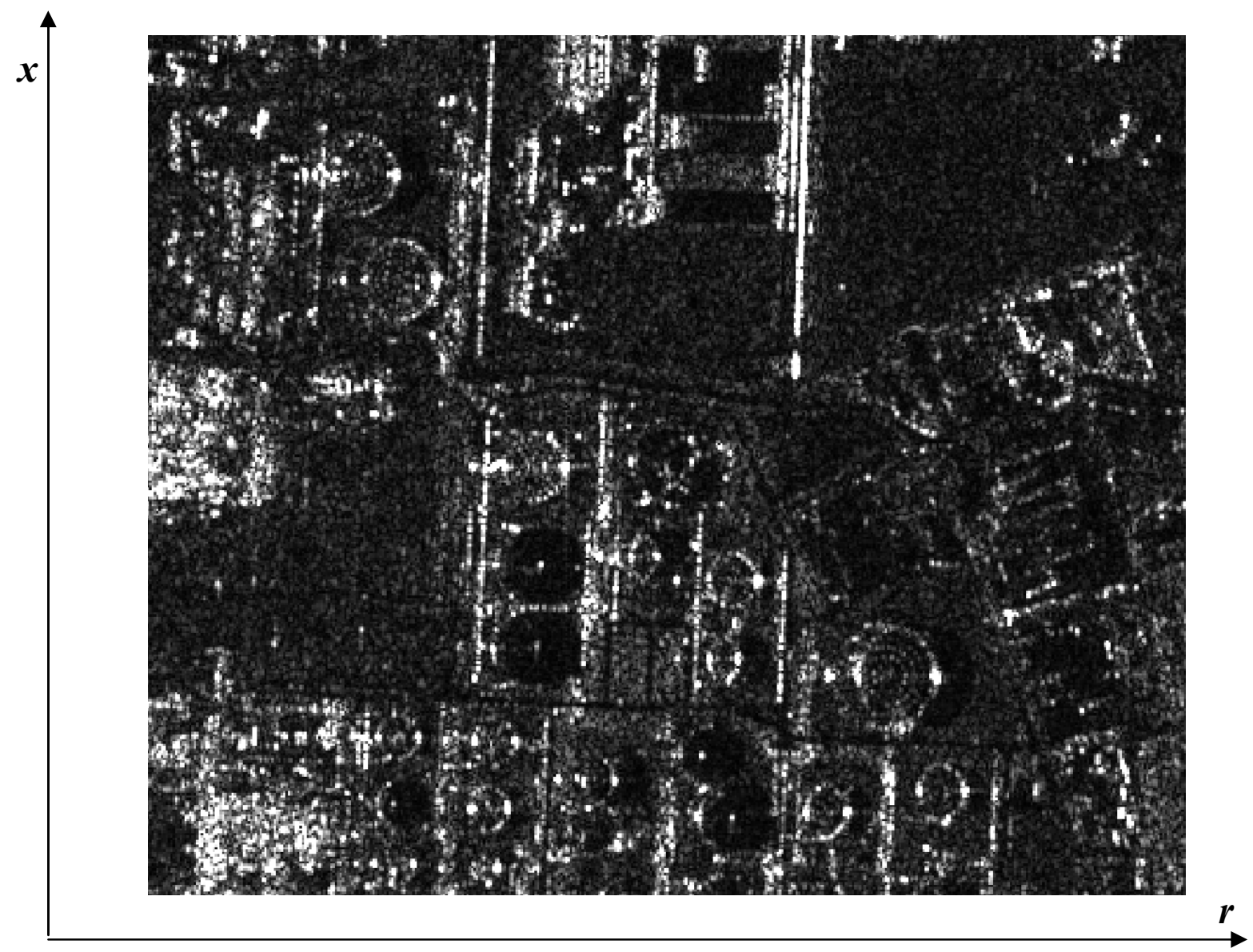

Figure 7

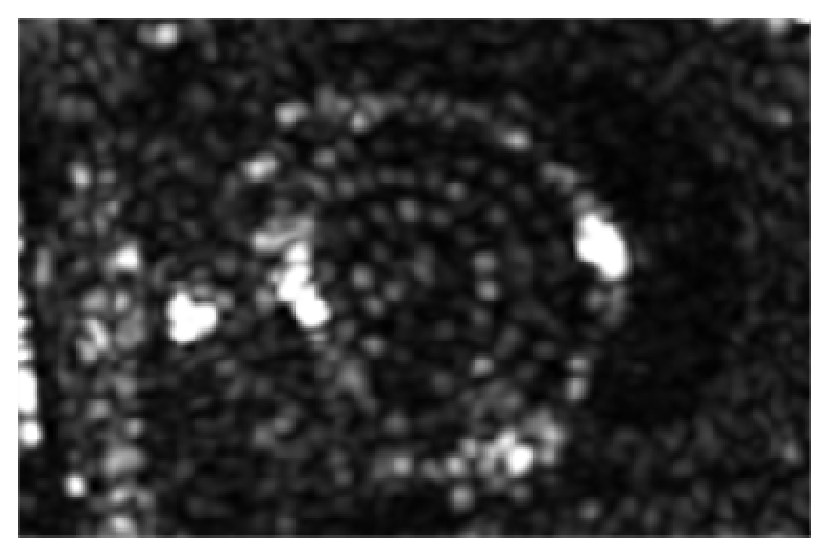

(a)

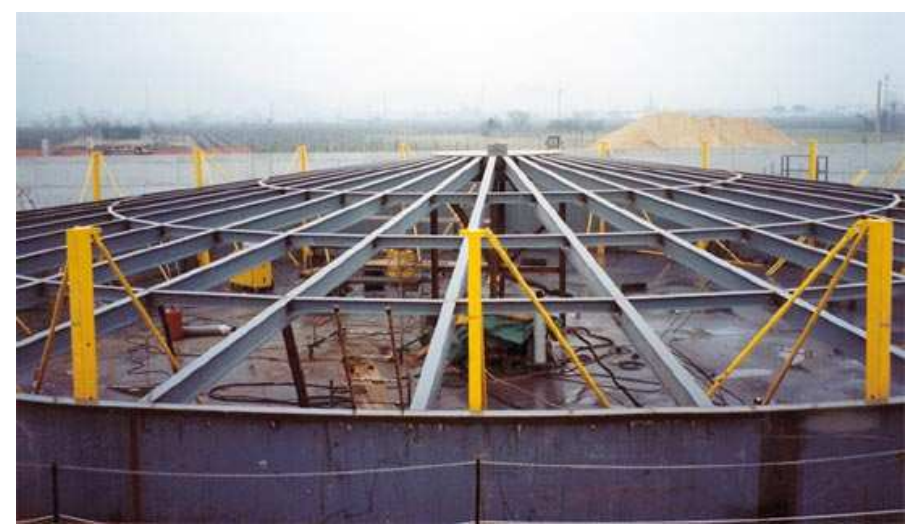

(b)

Figure 8 


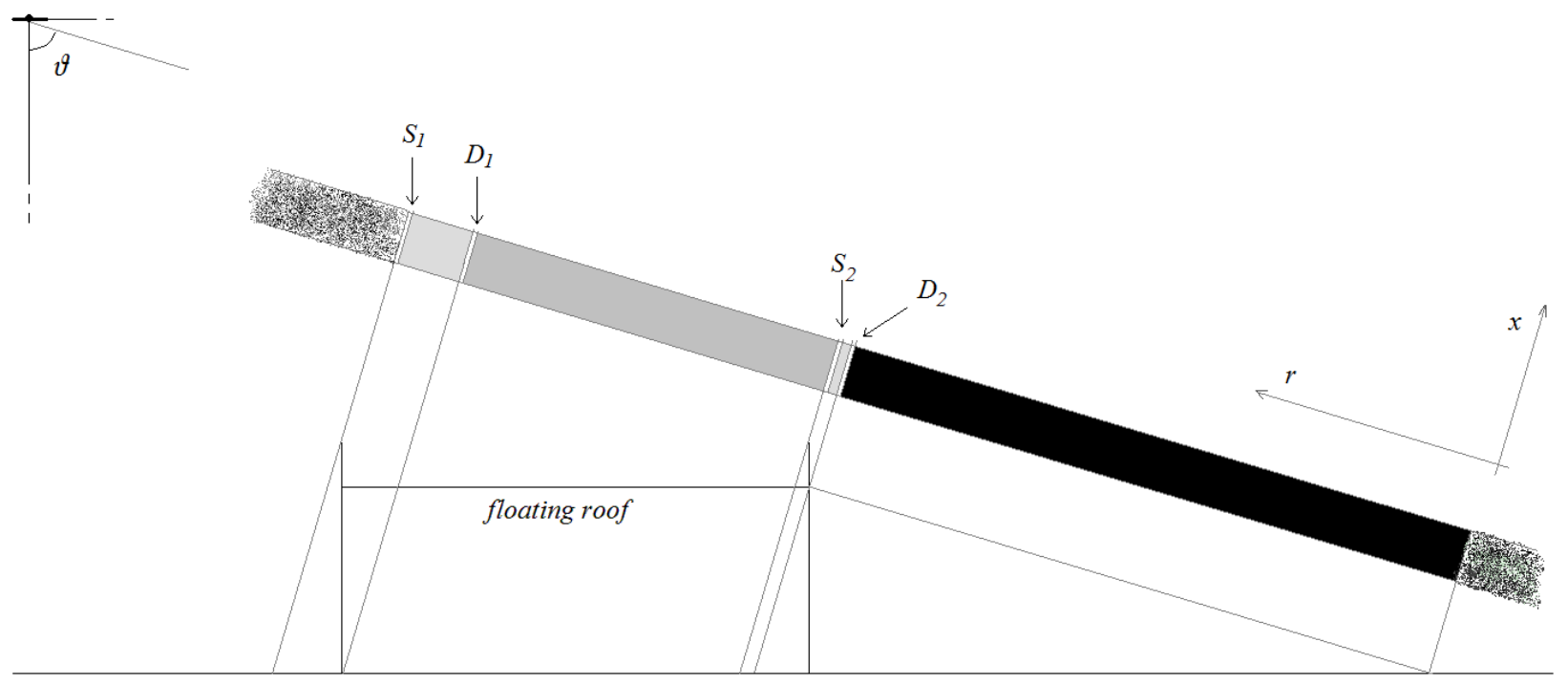

Figure 9

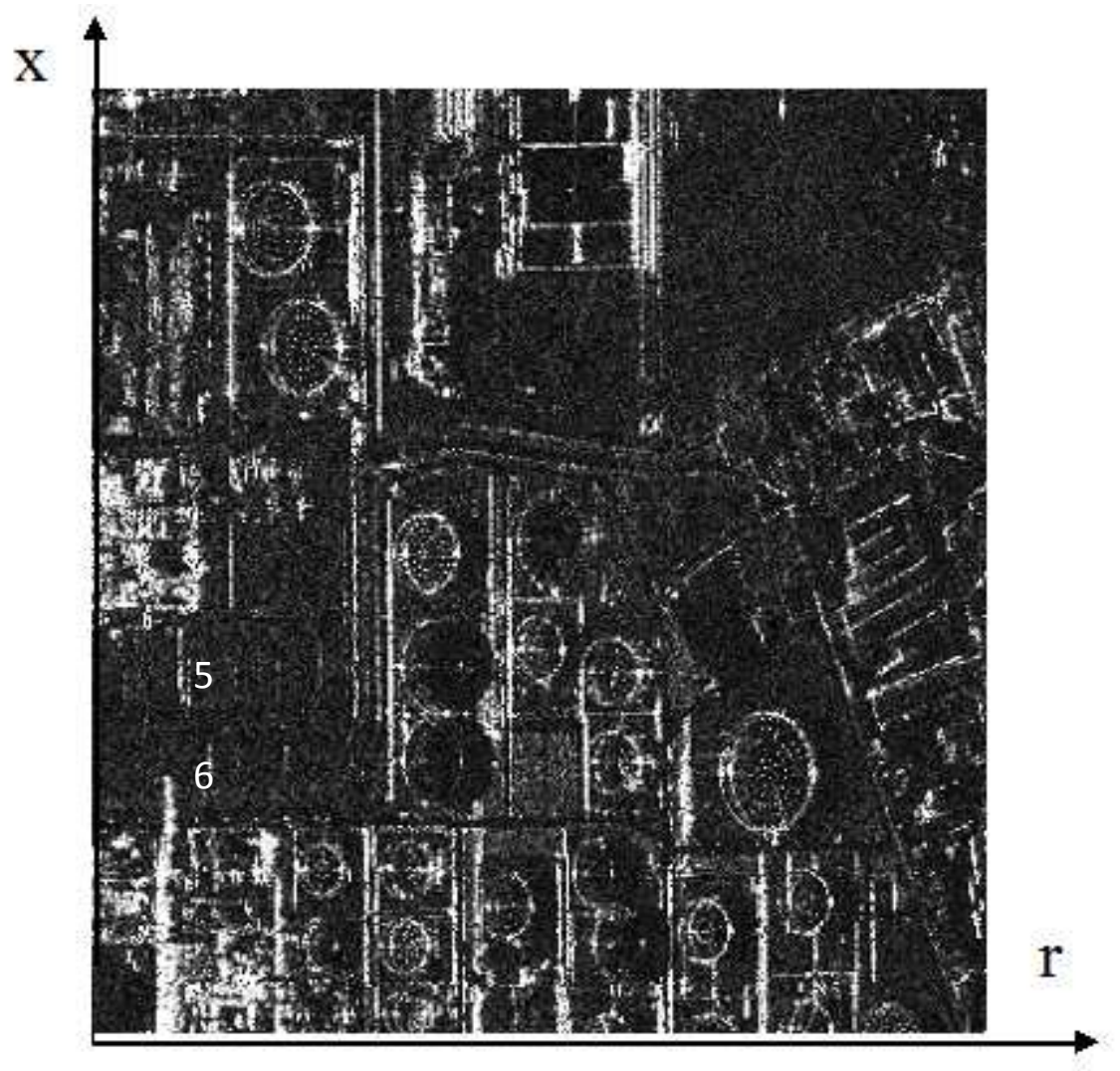

Figure 10 


\section{Captions of Tables}

Table I: Radar and image parameters for the products EEC_SE_SM, SSC_SM, SSC_HS.

Table II: Ground truth and diameter retrieval for the products EEC_SE_SM, SSC_SM, SSC_HS.

Table III: Ground truth and height retrieval for the products EEC_SE_SM, SSC_SM, SSC_HS. 
Table I

\begin{tabular}{|l|c|c|c|}
\hline Data Product & EEC_SE_SM & SSC_SM & SSC_HS \\
\hline Frequency & $9.65 \mathrm{GHz}$ & $9.65 \mathrm{GHz}$ & $9.65 \mathrm{GHz}$ \\
\hline Mode & Stripmap & Stripmap & High Spotlight \\
\hline Polarization & $\mathrm{HH}$ & $\mathrm{HH}$ & $\mathrm{HH}$ \\
\hline Look angle (scene center) & $29^{\circ}$ & $29^{\circ}$ & $54^{\circ}$ \\
\hline Azimuth resolution & $3,09 \mathrm{~m}$ & $3,00 \mathrm{~m}$ & $1,10 \mathrm{~m}$ \\
\hline Slant range resolution & - & $1,18 \mathrm{~m}$ & $1,17 \mathrm{~m}$ \\
\hline Ground range resolution & $3,04 \mathrm{~m}$ & - & - \\
\hline Azimuth Pixel spacing & $1,25 \mathrm{~m}$ & $1,90 \mathrm{~m}$ & $0,87 \mathrm{~m}$ \\
\hline Slant range Pixel spacing & - & $0,91 \mathrm{~m}$ & $0,91 \mathrm{~m}$ \\
\hline Ground range Pixel spacing & $1,25 \mathrm{~m}$ & - & - \\
\hline
\end{tabular}

Table II

\begin{tabular}{|c|c|c|c|c|c|c|c|c|c|c|c|}
\hline \multirow{2}{*}{\multicolumn{2}{|c|}{ Ground Truth }} & \multicolumn{10}{|c|}{ Feature Extraction } \\
\hline & & \multicolumn{2}{|c|}{ EEC Stripmap } & \multicolumn{4}{|c|}{ SSC Stripmap } & \multicolumn{4}{|c|}{ SSC Spotlight } \\
\hline N.Tank & $\begin{array}{c}\mathrm{D} \\
{[\mathrm{m}]}\end{array}$ & $\begin{array}{l}\mathrm{D}[\mathrm{m}] \\
\text { (from } \\
\text { range) }\end{array}$ & $\begin{array}{l}\left|\mathrm{e}_{\mathrm{D}}\right| \\
{[\mathrm{m}]}\end{array}$ & $\begin{array}{l}\mathrm{D}[\mathrm{m}] \\
\text { (from } \\
\text { range) }\end{array}$ & $\begin{array}{l}\left|\mathrm{e}_{\mathrm{D}}\right| \\
{[\mathrm{m}]}\end{array}$ & $\begin{array}{c}\mathrm{D}[\mathrm{m}] \\
\text { (from } \\
\text { azimuth) }\end{array}$ & $\begin{array}{l}\left|\mathrm{e}_{\mathrm{D}}\right| \\
{[\mathrm{m}]}\end{array}$ & $\begin{array}{l}\mathrm{D}[\mathrm{m}] \\
\text { (from } \\
\text { range) }\end{array}$ & $\begin{array}{l}\left|\mathrm{e}_{\mathrm{D}}\right| \\
{[\mathrm{m}]}\end{array}$ & $\begin{array}{c}\mathrm{D}[\mathrm{m}] \\
\text { (from } \\
\text { azimuth) }\end{array}$ & $\begin{array}{l}\left|e_{\mathrm{D}}\right| \\
{[\mathrm{m}]}\end{array}$ \\
\hline 1 & 84,73 & 91,68 & 7,2 & 88,12 & 3,39 & 87,40 & 2,67 & 85,39 & 0,66 & 84,10 & 0,63 \\
\hline 2 & 56,30 & 79,19 & 22,89 & 76,87 & 20,57 & 57,00 & 0,70 & 57,30 & 1,00 & 57,22 & 0,92 \\
\hline 3 & 66,50 & 76,49 & 9,99 & 76,87 & 10,37 & 68,40 & 1,90 & 73,03 & 6,53 & 68,49 & 1,99 \\
\hline 4 & 66,50 & 75,02 & 8,52 & 73,12 & 6,62 & 68,40 & 1,90 & 74,16 & 7,66 & 67,63 & 1,13 \\
\hline
\end{tabular}

Table III

\begin{tabular}{|c|c|c|c|c|c|c|c|c|c|c|c|c|}
\hline \multirow{2}{*}{\multicolumn{2}{|c|}{ Ground Truth }} & \multicolumn{11}{|c|}{ Feature Extraction } \\
\hline & & \multicolumn{3}{|c|}{ EEC Stripmap } & \multicolumn{4}{|c|}{ SSC Stripmap } & \multicolumn{4}{|c|}{ SSC Spotlight } \\
\hline N.Tank & $\mathrm{h}[\mathrm{m}]$ & $\begin{array}{c}\mathrm{h}[\mathrm{m}] \\
\text { from } \\
\text { geometry }\end{array}$ & $\begin{array}{l}\left|\mathrm{e}_{\mathrm{h}}\right| \\
{[\mathrm{m}]}\end{array}$ & $\begin{array}{c}\mathrm{h}[\mathrm{m}] \\
\text { from } \\
\text { radiometry }\end{array}$ & $\begin{array}{c}\mathrm{h}[\mathrm{m}] \\
\text { from } \\
\text { geometry }\end{array}$ & $\begin{array}{l}\left|\mathrm{e}_{\mathrm{h}}\right| \\
{[\mathrm{m}]}\end{array}$ & $\begin{array}{c}\mathrm{h}[\mathrm{m}] \\
\text { from } \\
\text { radiometry }\end{array}$ & $\begin{array}{l}\left|\mathrm{e}_{\mathrm{h}}\right| \\
{[\mathrm{m}]}\end{array}$ & $\begin{array}{c}\mathrm{h}[\mathrm{m}] \\
\text { from } \\
\text { geometry }\end{array}$ & $\begin{array}{l}\left|\mathrm{e}_{\mathrm{h}}\right| \\
{[\mathrm{m}]}\end{array}$ & $\begin{array}{c}\mathrm{h}[\mathrm{m}] \\
\text { from } \\
\text { radiometry }\end{array}$ & $\begin{array}{l}\left|e_{h}\right| \\
{[\mathrm{m}]}\end{array}$ \\
\hline 1 & 14,40 & 13,92 & 0,48 & NA & 14,55 & 0,15 & $\begin{array}{c}\text { NA } \\
\text { (calibrator) }\end{array}$ & - & 12,37 & 2,03 & $\begin{array}{c}\text { NA } \\
\text { (calibrator) }\end{array}$ & - \\
\hline 2 & 14,60 & 14,28 & 0,32 & NA & 15,59 & 0,99 & 14,55 & 0.05 & 15,46 & 0,86 & 14,35 & 0,25 \\
\hline 3 & 14,70 & 9,92 & 4,78 & NA & 10,39 & 4,31 & 14,16 & 0.54 & 15,46 & 0,86 & 14,39 & 0,31 \\
\hline $\begin{array}{l}4 \\
5 \\
6\end{array}$ & 14,70 & 14,01 & 0,69 & NA & 15,59 & 0,89 & $\begin{array}{c}\text { NA } \\
\text { (calibrator) }\end{array}$ & - & 12,37 & 2,33 & $\begin{array}{c}\text { NA } \\
\text { (calibrator) } \\
15,30 \\
14,24\end{array}$ & $\begin{array}{l}- \\
<1 \\
<1\end{array}$ \\
\hline
\end{tabular}

